\title{
EVOLUCION DE LOS PRECIOS DE LA TIERRA EN UN MERCADO LOCAL: LA COMARCA DEL CAMPO DE CARTAGENA (1866-1974)
}

CANDIDO ROMAN CERVANTES

Universidad de Murcia

\section{RESUMEN}

A través de la cuantificación de los datos de una fuente muy poco explorada como es el Archivo del Banco Hipotecario de España, he pretendido reconstruir el comportamiento de una variable esencial en toda estructura agraria: el precio de la tierra. Analizar sus fluctuaciones, así como su evolución a largo plazo, nos ofrece una perspectiva mucho más completa, puesto que pone de manifiesto su estrecha dependencia con las distintas coyunturas históricas. Por otra parte, aproximarnos a las cotizaciones unitarias de los diferentes cultivos hasta la actualidad era una manera de comprobar cuándo y por qué se producen los cambios de tendencia en sus respectivas valoraciones.

\section{ABSTRACT}

Through an examination of a source which has been little used to date, namely mortgages in the archives of the Banco Hipotecario de España, I have tried to measure changes in land prices. Both short and long term fluctuations are considered, and these show a close relationship to other economic variables. Finally, by looking at the experience of different agricultural commodities, we are able both to check, and explain, the changes in land prices.

Antes de abordar este tema debo hacer algunas consideraciones de tipo bibliográfico y también metodológico. Los estudios que hay en España sobre el precio de la tierra son por lo general muy escasos. Para el siglo XIX disponemos tan sólo, que sepamos, de los trabajos de Ricardo Robledo ', y el más reciente de María Parias Sainz de Rozas para tierras sevillanas. Sin embargo, en Francia e Italia tienen una mayor tradición al respecto, como quedó reflejado,

1 Robledo, R. (1983), pp. 253-275. 
entre otras publicaciones, en el monográfico que la revista Quaderni Storici (1987), número 65, dedicó al estudio del precio de la tierra durante los siglos XVIII y XIX. Pero si para la anterior centuria la producción científica española es escasa, para el siglo xx y hasta 1986 no disponíamos de trabajos de cierta envergadura. Estos quedaron plasmados en el número 41 de la revista Agricultura y Sociedad, donde se daban a conocer los resultados de la encuesta realizada en 1979 por la Secretaría General Técnica del Ministerio de Agricultura y los Servicios Centrales y Periféricos de las Comunidades Autónomas ${ }^{2}$. En dicho número se incluyen excelentes trabajos regionales como el de Consuelo Varela ${ }^{3}$, José María Sumpsi ${ }^{4}$, Eladio Arnalte Alegre y Lorenzo Avella Reus 5 , además de un fichero de referencias bibliográficas sobre el mercado de la tierra y sus efectos sobre la dinámica de las estructuras agrarias.

La mayoría de los autores anteriormente citados emplean como instrumentos de análisis modelos econométricos, por otra parte, muy propios de las técnicas de investigación en el campo de la economía agrícola. Fuera de él debo citar los trabajos en geografía agraria de José Costa Mas sobre el Bajo Segura ${ }^{6}$, el de Josefina Cruz Villalón relativo al Valle del Bajo Guadalquivir ${ }^{7}$ y el de Luis Molinero Hernando sobre Castilla la Vieja ${ }^{8}$.

En el área de la historia económica, y más concretamente en historia agraria, se ha avanzado muy poco por las dificultades de tipo heuristico que conlleva el seguimiento de una variable tan heterogénea como es el precio de la tierra a largo plazo. Reconstruir una serie temporal durante cien años tan sólo es posible si se recurre, como lo hizo Maria Parias, al vaciado sistemático de las escrituras de compraventas. Aun así, lo disperso de la información obliga a la selección de los datos más uniformes dando como resultado, a pesar de todo, unas cifras que en el mejor de los casos han de tomarse como estimaciones. Ahi radica quizá la dificultad de abordar estos estudios desde la perspectiva histórica, por otra parte imprescindible para saber cómo se ha estructurado la oferta y demanda del mercado de tierras en una zona y periodo determinado. Los estudios de economía agraria a que aludía anteriormente manejan una información actual y de fácil accesibilidad, donde el análisis retrospectivo a largo

2 Sánchez Rodriguez, P. (1986), pp. 204.205.

3 Varela Ortega, C. (1986a), pp. 73-137. También de la misma autora (1988), pp. 231-270.

${ }^{4}$ Sumpsi Viñas, J. M." (1986), pp. 15-72. Una versión anterior de este trabajo puede verse en Papeles de Economía Española, 1983, n. ${ }^{\circ} 16$, Madrid, Confederación Española de Cajas de Ahorro, pp. 169-182.

s Arnalte Alegre, E., y Avella Reus, L. (1986), pp. 139-183.

6 Costa Mas, J. (1981), pp. 419-436.

7 Cruz Villalón, J. (1981), pp. 241-252.

* Molinero Hernando, L. (1981), pp. 253-265. 
plazo no forma parte de su metodología. Por ello, creo que es misión del historiador de la economía el afrontar dicho reto.

\section{CONSIDERACIONES PREVIAS}

Habitualmente el precio de la tierra ha estado unido a la cantidad de renta que producía y por tanto ahí se ha buscado el único factor causante de sus fluctuaciones 9. Por ello, ante coyunturas donde el precio de los productos agricolas experimentaban un fuerte aumento, éste iba acompañado con el de la tierra. En realidad la teoría ricardiana de la renta se basa en la tierra como factor de producción, por lo que la renta obtenida se considera como fruto de las potencialidades productivas del suelo agrario. Durante la Edad Moderna dicha conexión mediatizaba las relaciones de producción en aquellas comunidades cuya actividad económica fundamental era la agricultura. Un período de malas cosechas llevaba implícito un encarecimiento de los productos agrícolas $\mathrm{y}$, por consiguiente, un aumento de la demanda sobre el mercado de tierras que incrementaba su precio, haciéndolo inaccesible para la mayoría de los campesinos. Pero si esto podría venir por el lado de la demanda, por el de la oferta podría producirse una bajada en la cotización al concurrir un elevado número de explotaciones como consecuencia del grado de endeudamiento y, por tanto, de las necesidades monetarias de los agricultores ${ }^{10}$.

La elasticidad en el precio del factor tierra ha estado y está, aunque en menor medida, vinculada con las fluctuaciones del valor de los cultivos. Un trabajo reciente dedicado al estudio de la evolución del precio de la tierra en Francia de 1850 a 1980 llegaba, entre otras, a las siguientes conclusiones:

«valeur vénale et valeur locative sont à la hausse quand le produit net par hectare augmente fortement (1850-1880), puis une baisse s'amorce avec la crise agricole qui va, grosso modo, se prolonger jusqu'en 1914» ${ }^{11}$; en el mismo sentido se afirma: «la crise de 1880 montre bien que, dans les regions d'agriculture marchande, le prix des terres a suivi le prix des denrées principales - blé, vin, viande-, s'effondrant quand ces derniers s'affaissent, résistant mieux ailleurs. Nous montrerons qu'il en est de même aujourd'hui» ${ }^{12}$.

9 Varela Ortega, C. (1986a), p. 210.

${ }_{10}$ Sclarandis, C. (1987), pp. 467-492.

1 Boinon, J. P., y Cavailhès, J. (1988), p. 217.

12 Boinon, J. P., y Cavailhès, J. (1988), p. 219. 
Parece ser que no hay duda sobre el efecto regulador de los cultivos en el precio de la tierra, pero debemos plantearnos si éste debe sus fluctuaciones dependiendo sólo y exclusivamente de dicha variable. El tamaño de la explo. tación, la calidad de las tierras, las rentas de localización y lo que se podría calificar como «imponderables» ${ }^{13}$, es decir, estimaciones personales basadas en el capricho de los compradores y vendedores, también van a incidir en la valoración final. Pero si las causas antes descritas podrían limitarse tan sólo a aspectos localizados en el ámbito concreto de la explotación, también deben tenerse en cuenta los factores de tipo macroeconómico y coyuntural. Por ejemplo, la importante movilidad y subida de precios durante la década de los años sesenta y setenta debido a las elevadas tasas de inflación hizo de la tierra un valor refugio para aquellos capitales procedentes de fuera del sector ${ }^{14}$. Bien para especular o bien para su uso agricola, la década de los setenta se convierte en un periodo de «faim de terres».

La crisis del petróleo, la escasa rentabilidad del ahorro en la banca, la pérdida de poder adquisitivo del dinero, así como la inestable situación de la industria serán algunas de las causas determinantes en el aumento de la demanda de tierras en un periodo de grandes inversiones, la mayoría de ellas con objetivos claramente especulativos. Como ha señalado Naredo, «la mayor parte de los capitales que se dirigen a la agricultura buscan más asegurar plusvalías derivadas de la revalorización de la tierra, que rentas procedentes de su explotación» ${ }^{15}$. Pero si los motivos aludidos pueden ser los causantes de una subida del valor venal y también locativo de la tierra a nivel nacional, en la Comarca del Campo de Cartagena se da, como más adelante veremos, un elemento añadido que va a justificar aún más dicha tendencia: las perspectivas de ampliación de los regadios por el Trasvase Tajo-Segura a partir de 1972.

Quisiera finalizar estas breves reflexiones introductorias insistiendo una vez más sobre la correlación que hay entre la renta y el precio de la tierra, como variable esencial a la hora de fijar la mayoria de las transacciones territoriales, teniendo en cuenta, no obstante, que dicha correlación tiende a romperse en la actualidad: «el precio de la tierra acelera su crecimiento cuando los precios agrarios observan una mayor moderación. Las tierras con menor rendimiento agronómico (los pastizales, susceptibles sólo de aprovechamientos ganaderos o cinegéticos extensivos) son, paradójicamente, las que más han subi-

\footnotetext{
13 Parias, M." (1989), p. 161.

14 Sumpsi Viñas, J. M." (1986), p. 47.

1s Naredo, J. M. (1989), mimeo.
} 
do de precio» ${ }^{16}$. Así pues, la tierra se convierte en un activo dejando de ser sólo, y exclusivamente, un factor de producción ${ }^{17}$.

\section{FUENTES Y METODOLOGIA}

Es mi propósito estimar la evolución del precio de la tierra en la Comarca del Campo de Cartagena, su valor unitario y cómo éste ha ido fluctuando a lo largo del periodo de estudio. En cuanto al espacio cronológico, pretendo abarcar el mayor número de años posibles, dependiendo claro está de la información disponible. Por su variada procedencia, la confección del material empírico se ha realizado atendiendo a aquellos criterios que más posibilitaban rentabilizar la documentación. Aunque el estudio finaliza con los datos de algunos cultivos en 1990 a escala regional, he agrupado las fuentes buscando la confección de series temporales completas. La más extensa es la realizada con los datos sobre precios unitarios de secano-cereal procedentes de los expedientes de la Sección de Préstamos Agrícolas del Archivo del Banco Hipotecario de España (A.B.H), la cual informa sobre la valoración de la hectárea de tierra en la fecha de concesión del préstamo. Aunque dispongo de cifras desde 1882 a 1970, he tenido que seleccionarlas atendiendo a criterios de eficacia operativa, limitándome al período 1901-1962. Así es, como se verá más adelante al comentar los resultados, hay años donde he desechado aquella información que podia distorsionar la serie. Por ejemplo, para los años 1962-1970 tenía referencias sobre precios unitarios de regadio, cuyos datos no han podido ser empleados por lo irrevelante de su número. Por ello, me vi obligado, con la intención de simplificar aún más los cálculos, a homogeneizar las cifras que hacian referencia a tierras de secano-cereal. Del mismo modo, no incluí en el estudio los precios relativos al arbolado de secano por ser muy discontinuos y tener grandes lagunas. Era absurdo intentar reconstruir una serie de precios por aprovechamientos cuando la fuente no lo posibilitaba.

Así pues, reuní los años más completos y procedí a cuantificarlos. En primer lugar, no en todos hay el mismo número de observaciones; podía darse el caso, como en el período 1946-1960, donde éstas superan las 11 anuales, y

16 Naredo, J. M. (1989), ibidem.

17 Como señaló Varela, C. (1986b), p. 212, en Estados Unidos los analistas observan cómo el precio de la tierra se distancia de la renta agraria. Las causas fueron «... la demanda de tierras para usos no agrarios, la política de sostenimiento de precios y el avance tecnológico. Este último como responsable del exceso de capacidad de las explotaciones y, por tanto, de la presión de la demanda de tierras para ampliación de las mismas..... 
otros, como el de 1901-1910, que están entre 3 y 5 . En todas he aplicado el promedio aritmético, porque pensé que era el modo más idóneo de aproximar magnitudes. Como es obvio, hay años donde no se realizaron préstamos y por tanto carezco de cifras. En estos casos, y con el ánimo de no romper la serie, estimé los precios corrientes proyectando la media sobre los dos precios correlativos. Con esto, y exceptuando los años de la contienda civil 1936-1939, obtuve un serie bastante ajustada.

Habida cuenta de las importantes tasas de inflación del período 1940-1951, con un porcentaje para este último año del 28.3 por ciento ${ }^{18}$, era necesario proceder a deflactar los precios y pasarlos a precios constantes. Para ello utilicé como índice deflactor el obtenido a partir de los datos que incorpora la serie de la Renta Nacional estimada por Angel Alcaide Inchausti ${ }^{19}$. Con dichos indices obtuve las pesetas constantes convirtiendo la serie a números índices. También creí conveniente incorporar las tasas de variaciones interanuales aplicadas por igual tanto a los precios corrientes como a los constantes, detectando de este modo en qué años se producen las fluctuaciones más significativas.

Para poder reconstruir los precios por aprovechamientos tuve que recurrir a fuentes de muy diversa procedencia. La heterogeneidad de éstas no invalida los datos que he obtenido, ya que, como más adelante explicaré, son merecedoras de toda confianza. Para 1866 utilizo los datos del Consulado de Francia en Cartagena (A.C.F.C) a partir de las contestaciones al "Interrogatorio» sobre el estado de la agricultura en la Región de Murcia. En su respuesta informa de la valoración de las tierras de cereales, olivo, viña y huerta en el término municipal de Cartagena ${ }^{20}$. Para fines del siglo XIX, 1886, dispongo de las informaciones de los registradores de la propiedad, Memorias y Estados formados por los Registradores de la Propiedad en cumplimiento de lo prevenido en el R.D de 31 de agosto de 1886, que contienen datos sobre «el valor medio de una hectárea de terrenom en cereal, olivo, viña y huerta ${ }^{21}$.

Ya en la actual centuria, he podido cubrir el periodo 1900-1940 gracias al Archivo de Protocolos Notariales de Cartagena-Fuente Alamo (A.P.N.C). Los sondeos decenales pueden cubrir prácticamente el comportamiento del precio de la tierra durante el primer tercio del siglo $\mathrm{xx}$, incluyendo además las tendencias alcistas a partir del año 1940. Para la segunda mitad del siglo cuento con las cifras del Archivo de la Cámara Agricola de Cartagena (A.C.A.C) de 1965, relativas

18 González, M. J. (1990), p. 22.

19 Alcaide, A. (1976), pp. 1127-1150.

20 Archivo del Consulado de Francia en Cartagena, Libro 6, pregunta 5."

21 Como indicó Robledo, R. (1983), p. 274, «Para tal fin, los registradores necesitaron acudir a las oficinas de administración, ayuntamientos, comisiones de evaluación y peritos agrónomos». 
al término municipal de Cartagena. Se trata de la distribución de valores unitarios por cultivos propuestos por el Servicio Provincial del Catastro. Como la información venía segregada por calidades de suelos, agrupé los datos obteniendo el promedio entre los distintos grupos. La fuente especifica el producto bruto, el valor en venta, la renta rústica antes de impuestos, el interés y beneficio del cultivo, el tipo evaluatorio y por último el precio en el momento del informe. A pesar de señalar los valores según el tipo de riego, muestro una síntesis simplificada donde incluyo los precios de secano y los de regadío, estos últimos con «agua propia», por entender que los de «agua comprada» no eran representativos de los valores que estaban resultando a lo largo del período de análisis. No he encontrado más información válida hasta 1974, año en el que se aprueba el Decreto del Plan General de Transformación de las Zonas Regables del Campo de Cartagena, en las provincias de Murcia y Alicante, tras la declaración en 1972 de «Zona de Alto Interés Nacional». En su capítulo III se fijan los precios máximos y mínimos en secano y regadío, según la calidad de las tierras y cultivos.

Por último, completa el muestreo los precios facilitados por la Sección de Estadística de la Consejería de Agricultura, Ganadería y Pesca de la Comunidad Autonóma de la Región de Murcia para el periodo 1981-1990. A pesar de los intentos, no ha sido posible obtener información desagregada por comarcas, por lo que utilizaré datos regionales. Estos pueden servir en algunos cultivos como cereales y arbolado de secano,ya que conservan, grosso modo, unos valores muy similares en el conjunto de la región. No obstante, son los únicos datos de que dispongo para fechas recientes, lo cual puede ser de utilidad para conocer cuáles son las tendencias actuales en la cotización de los cultivos.

\section{ANALISIS DE LOS RESULTADOS}

Antes de entrar de lleno en el comentario del presente cuadro es preciso señalar dos cuestiones. La primera hace referencia a la elección del deflactor implícito, y la segunda, a la propia validez de los resultados y a su nivel de representatividad en la evolución del precio de las tierras de secano-cereal en la comarca. En cuanto al primer punto, la elección de la estimación hecha por Alcaide a partir de la contabilidad nacional me servía por cuanto abarcaba la totalidad del periodo donde tenía depositada la mayor parte de la información estadistica. En efecto, los «Informes Económicos Anuales» que publica el Servicio de Estudios del Banco de Bilbao, por su carácter no oficial también constituyen una buena alternativa a la hora de deflactar los precios - como lo 
demuestra su frecuente utilización - ${ }^{22}$; sin embargo, la ausencia de datos para el período 1900-1954, limitaba a priori la confección de la serie.

\section{CUADRO 1}

Evolución de los precios medios unitarios de la tierra de secano-cereal en la Comarca del Campo de Cartagena. Años 1901-1962

\begin{tabular}{|c|c|c|c|c|c|c|}
\hline$A$ ños & $I$ & $I I$ & $I I I$ & $I V$ & $V$ & $V I$ \\
\hline 1901 & 388 & 100,00 & 388 & 100,00 & - & - \\
\hline 1902 & 401 & 103,05 & 389 & 100,25 & 3,3 & 0,3 \\
\hline 1903 & 420 & 106,11 & 396 & 106,11 & 4,7 & 1,8 \\
\hline $1904 \ldots \ldots$ & 431 & 109,15 & 395 & 101,80 & 2,6 & $-0,3$ \\
\hline $1905 \ldots \ldots$ & 490 & 112,20 & 437 & 112,62 & 13,7 & 10,6 \\
\hline $1906 * \ldots$ & 513 & 115,32 & 445 & 114,69 & 4,7 & 1,8 \\
\hline $1907 * \ldots \ldots$ & 535 & 116,91 & 458 & 118,04 & 4,3 & 2,9 \\
\hline $1908 * \ldots \ldots$ & 558 & 119,82 & 466 & 120,10 & 4,3 & 1,7 \\
\hline $1909 \ldots \ldots$ & 580 & 123,64 & 469 & 120,87 & 4,0 & 0,6 \\
\hline $1910 \ldots \ldots$ & 601 & 122,03 & 492 & 126,80 & 3,6 & 4,9 \\
\hline $1911 \ldots \ldots$. & 611 & 130,25 & 469 & 120,87 & 1,7 & $-4,7$ \\
\hline $1912 \ldots \ldots$ & 647 & 127,98 & 506 & 130,41 & 5,9 & 7,7 \\
\hline 1913 & 671 & 131,73 & 509 & 131,18 & 3,7 & 0,8 \\
\hline 1914 & 697 & 140,36 & 497 & 128,09 & 3,9 & $-2,4$ \\
\hline $1915 \ldots$. & 712 & 139,62 & 510 & 131,44 & 2,2 & 2,4 \\
\hline 1916 * $\ldots$. & 716 & 143,14 & 500 & 128,86 & 0,6 & $-1,9$ \\
\hline $1917^{*} \ldots$ & 720 & 154,80 & 465 & 119,84 & 0,6 & $-7,0$ \\
\hline $1918 * \ldots \ldots$ & 724 & 144,66 & 500 & 128,86 & 0,6 & 7,5 \\
\hline 1919 ..................... & 728 & 146,19 & 498 & 128,35 & 0,6 & $-0,4$ \\
\hline $1920 \ldots \ldots \ldots \ldots \ldots \ldots \ldots$ & 702 & 148,12 & 474 & 122,16 & $-3,6$ & $-4,8$ \\
\hline $1921 \ldots \ldots \ldots \ldots \ldots \ldots \ldots \ldots$ & 710 & 146,96 & 483 & 124,48 & 1,1 & 1,8 \\
\hline $1922 \ldots \ldots \ldots \ldots \ldots \ldots \ldots \ldots$ & 667 & 149,67 & 446 & 114,94 & $-6,0$ & $-7,6$ \\
\hline $1923 \ldots \ldots \ldots \ldots \ldots \ldots \ldots \ldots$ & 673 & 153,45 & 439 & 113,14 & 0,9 & $-1,5$ \\
\hline $1924 \ldots \ldots$ & 680 & 156,98 & 433 & 111,59 & 1,0 & $-1,3$ \\
\hline 1925 & 685 & 168,85 & 406 & 104,63 & 0,7 & $-6,2$ \\
\hline 1926 & 691 & 172,52 & 401 & 103,35 & 0,9 & $-1,2$ \\
\hline $1927 \ldots \ldots \ldots \ldots \ldots$ & 757 & 182,48 & 415 & 106,95 & 9,6 & 3,4 \\
\hline $1928 \ldots \ldots \ldots \ldots \ldots$ & 788 & 186,95 & 422 & 108,76 & 4,1 & 1,6 \\
\hline $1929 \ldots \ldots$. & 864 & 187,14 & 462 & 119,07 & 9,6 & 9,4 \\
\hline $1930 \ldots \ldots \ldots$ & 900 & 190,71 & 472 & 121,64 & 4,2 & 2,1 \\
\hline $1931 \ldots \ldots \ldots \ldots \ldots \ldots \ldots$ & 989 & 199,33 & 496 & 127,83 & 9,9 & 5,0 \\
\hline
\end{tabular}

22 Entre otros, Garcia González, A., y Barciela López, C. (1986), 508-509. También Domingo et al. (1987), p. 19. 


\section{CUADRO 1 (continuación)}

Evolución de los precios medios unitarios de la tierra de secano-cereal en la Comarca del Campo de Cartagena. Años 1901-1962

\begin{tabular}{|c|c|c|c|c|c|c|}
\hline Años & $I$ & II & III & IV & $V$ & $V I$ \\
\hline 1932 & 975 & 201,40 & 484 & 124,74 & $-1,3$ & $-2,4$ \\
\hline $1933 \ldots .$. & 993 & 199,33 & 498 & 128,35 & 1,8 & 2,8 \\
\hline $1934 * \ldots$ & 997 & 209,32 & 476 & 122,68 & 0,4 & $-4,4$ \\
\hline 1935 *. & 999 & 205,32 & 487 & 125,51 & 0,2 & 2,3 \\
\hline$\ldots \ldots \ldots \ldots \ldots \ldots$ & - & - & - & - & - & - \\
\hline $1937 \ldots \ldots .$. & - & - & - & - & - & - \\
\hline $1938 \ldots \ldots \ldots \ldots \ldots \ldots \ldots \ldots$ & - & - & - & - & - & - \\
\hline $1939 \ldots \ldots \ldots \ldots \ldots \ldots \ldots \ldots$ & - & - & - & - & - & - \\
\hline $1940 \ldots$ & 1.000 & 152,40 & 656 & 169,07 & 0,7 & 34,7 \\
\hline $1941 * \ldots$ & 1.188 & 154,47 & 769 & 198,19 & 18,8 & 17,2 \\
\hline $1942 \ldots \ldots$ & 1.375 & 165,43 & 831 & 214,17 & 15,7 & 8,0 \\
\hline 1943 * & 1.688 & 167,98 & 1.005 & 259,02 & 22,7 & 20,9 \\
\hline $1944 \ldots$ & 2.000 & 169,18 & 1.182 & 304,63 & 18,4 & 17,6 \\
\hline 1945 & 2.200 & 165,29 & 1.331 & 343,04 & 10,0 & 12,6 \\
\hline 1946 & 2.500 & 183,48 & 1.363 & 351,28 & 13,6 & 2,4 \\
\hline 1947 & 2.942 & 186,78 & 1.575 & 405,92 & 17,6 & 15,5 \\
\hline 1948 & 3.000 & 185,61 & 1.616 & 416,49 & 1,9 & 2,6 \\
\hline 1949 & 3.250 & 187,60 & 1.732 & 446,39 & 8,3 & 7,1 \\
\hline $1950 \ldots$ & 3.687 & 197,14 & 1.870 & 481,95 & 13,4 & 7,9 \\
\hline $1951 \ldots$ & 3.701 & 218,65 & 1.693 & 436,08 & 0,3 & $-9,4$ \\
\hline 1952 & 3.750 & 233,46 & 1.606 & 413,91 & 1,3 & $-5,1$ \\
\hline $1953 \ldots$ & 3.859 & 236,80 & 1.630 & 420,10 & 2,9 & 1,4 \\
\hline 1954 *. & 3.924 & 253,79 & 1.546 & 398,45 & 1,6 & $-5,1$ \\
\hline $1955 \ldots$ & 3.988 & 265,90 & 1.500 & 386,59 & 1,6 & $-2,9$ \\
\hline $1956 \ldots$ & 4.250 & 285,17 & 1.490 & 384,02 & 6,5 & $-0,6$ \\
\hline 1957 *. & 4.313 & 295,90 & 1.457 & 375,51 & 1,4 & $-2,2$ \\
\hline $1958 *$. & 4.375 & 311,18 & 1.406 & 362,37 & 1,4 & $-3,5$ \\
\hline 1959 *. & 4.438 & 302,82 & 1.466 & 377,83 & 1,4 & 4,2 \\
\hline $1960 \ldots$ & 4.500 & 306,83 & 1.467 & 378,09 & 1,3 & 0,0 \\
\hline 1961 & 5.100 & 344,78 & 1.479 & 381,18 & 13,3 & 0,8 \\
\hline 1962 & 5.825 & 379,73 & 1.534 & 395,36 & 14,2 & 3,7 \\
\hline
\end{tabular}

Años en los que no poseo datos. He estimado la media aritmética a partir de las cifras correspondientes a los años sucesivos.

Fuente: I. A.B.H, precios en pesetas corrientes secano-cereal.

II. Indice deflactor implícito (base 1901:100), a partir cional de España. Alcaide, A (1976), pp. 1142-1143.

III. Precios deflactados, pesetas constantes.

IV. Números índices en pesetas de 1901.

V. Tasas de variación anual (AV) a precios corrientes.

VI. Tasas de variación anual (AV) a precios constantes. 
En cuanto al grado de validez de las cifras puedo considerarlo de alto. Teniendo en cuenta las posibles desviaciones que pudieran haber entre las distintas zonas de la comarca (sectores oriental y occidental), pensé que la confección de un precio medio ponderado seria lo suficientemente indicativo del valor de la tierra. Es más, las diferencias podrían venir fundamentalmente por la intensidad de los cultivos (secano-regadio), cuestión que queda descartada al procesar la información sólo para el secano-cereal. Por otra parte, en espacios agroclimáticos relativamente reducidos como los del territorio que aquí nos ocupa la afinidad edafológica de los suelos suele ser alta, lo que aminora si cabe aún más la existencia de grandes diferencias en las valoraciones.

La referencia más lejana sobre precios unitarios de secano por calidades de suelos proviene de 1866, que fija en 221 ptas. las de $1 .{ }^{a}, 176$ ptas. las de $2 .^{a}$ y en 18 y 9 ptas. las de $3 .^{a}$ y $4 .^{a}$, respectivamente. Según el cónsul francés, «los precios de la tierra han subido considerablemente desde hace 30 años. Desde hace 15 años los propietarios rurales tienen asegurado el doble o el triple del valor de la tierra» ${ }^{23}$. Dicho comentario es lo suficientemente elocuente sobre el comportamiento alcista que estaban experimentando las tierras de labor-secano a mediados del siglo XIX. Cotejando estas cifras con las que expone Ricardo Robledo en el trabajo antes citado, para estos años se observa una cierta similitud de los valores con las tierras de secano en Valladolid, ya que éstas oscilan entre las 167 y 174 ptas./has ${ }^{24}$. Sin embargo, es en 1886 cuando el informe de los registradores de la propiedad atribuye, sin especificación de calidad, un valor medio unitario de 600 ptas. ${ }^{25}$. En veinte años se ha producido un incremento en relación a las tierras de $2{ }^{a}$ en torno al 71 por ciento, tasa que debe admitirse con precaución. No obstante, los datos proporcionados por los registradores de la propiedad para tierras castellanas fluctúan en magnitudes similares, «en 1886, más de las cuatro quintas partes de los datos habian superado las 200 pesetas, centrándose un 50 por 100 de la información entre las $300-750$ ptas.» ${ }^{26}$.

Si durante la segunda mitad del siglo XIX se llevan a cabo gran número de medidas liberalizadoras de la propiedad —entre ellas el fenómeno desamortizador-, que iban a propiciar la salida al mercado de un importante volumen de tierras y en consecuencia un incremento de la oferta, ¿por qué continuaba ascendente el precio de la tierra para las últimas décadas del siglo? En contra

${ }^{23}$ Archivo del Consulado de Francia en Cartagena, Libro 6, «Interrogatorio de 1866».

24 Robledo, R. (1983), p. 260.

25 Memorias y Estados formados por los Registradores de la Propiedad (1890), Vol. 4, Madrid.

26 Robledo, R. (1983), p. 261. 
de las leyes de la libre concurrencia y a pesar de la oferta masiva de tierras ${ }^{27}$, éstas incrementaron su precio. Ricardo Robledo relaciona dicha tendencia con la reducción de los costos de producción de la industria, así como por los adelantos en los transportes (sobre todo el ferrocarril), incidiendo en última instancia en una mejora en las fases de comercialización de los productos agrarios. Es decir, aumenta el valor de la tierra como factor de producción y, por consiguiente, incrementa su precio unitario.

No obstante, volviendo a la estimación para el Campo de Cartagena de 1886 me parece elevada si se la compara con la atribuida al resto del país para ese mismo año, 377 ptas./has. ${ }^{28}$. Si tuviera que buscar una explicación, ésta la encontraría en la muy probable inflación de precios que ocasionaba las excelentes rentas de situación que suponía la proximidad de los centros mineros. Estos incrementaban la renta per cápita de la zona no solamente en lo que respecta a los propietarios de minas que llegaron a acumular grandes fortunas in virtiéndolas en la agricultura, sino también en lo que respecta a los trabajadores que incrementaban sus tasas de liquidez monetaria pudiendo acceder al mercado de tierras.

Ha llegado el momento de relacionar los datos del Cuadro 1 con lo que estoy comentando. Es evidente que las diferencias en pesetas corrientes de 1901 (388 ptas.), respecto a la cifra que manejaba para 1886, indica que ha habido en términos generales una caída en la cotización en torno a un 35.33 por ciento. Veamos cuál podría ser la explicación. Como es sabido, la crisis finisecular que afectó a toda Europa fue sobre todo una crisis cerealícola. Es decir, aunque repercutió también en la rentabilidad de otros cultivos, propició el desplome en la cotización de los precios de los cereales, con la consiguiente baja de las rentas de las tierras dedicadas a su aprovechamiento. Tan sólo en este contexto es donde puede encontrarse la justificación del descenso, teniendo que esperar hasta 1910 para volver a alcanzar los valores de 1886.

Voy a centrarme en el análisis de los datos relativos a los números índices en pesetas constantes de 1901. La elección de 1901 como año base-100 se explica por constituir mi principal objetivo conocer cuál ha sido la evolución histórica de la cotización de las tierras. En cuanto a la utilidad de la tasas de variación interanual, radica en que miden con más precisión donde se producen las fluctuaciones más bruscas. En los 62 años que incluyo en la serie deben senalarse fases de distinta intensidad. A una etapa de alza (1905-1912)

27 Se calcula en torno a 10.000.000 de hectáreas la superficie vendida en España entre 1836 y 1900. Simón Segura, F. (1973), p. 282.

${ }_{28}$ Robledo, R. (1983), p. 269. 
le sigue otra donde el comportamiento de los precios oscila con altibajos (1913-1921), y anuncia un período de caidas (1922-1928), para ir remontando sus índices hasta 1935, pero sin alcanzar los valores del período 1905-1912. Tras la pausa de la contienda civil (1936-1939), la evolución de los precios tiene un sostenido comportamiento alcista, siendo en la década 1940-1950 donde se experimentan las subidas más espectaculares de toda la serie. Precisamente a lo largo de la década de los cincuenta, las cotizaciones moderan sus índices, produciéndose bajas significativas, para comenzar a subir en los dos únicos años de los sesenta.

\section{GRAFICO 1}

Precios medios unitarios (secano)

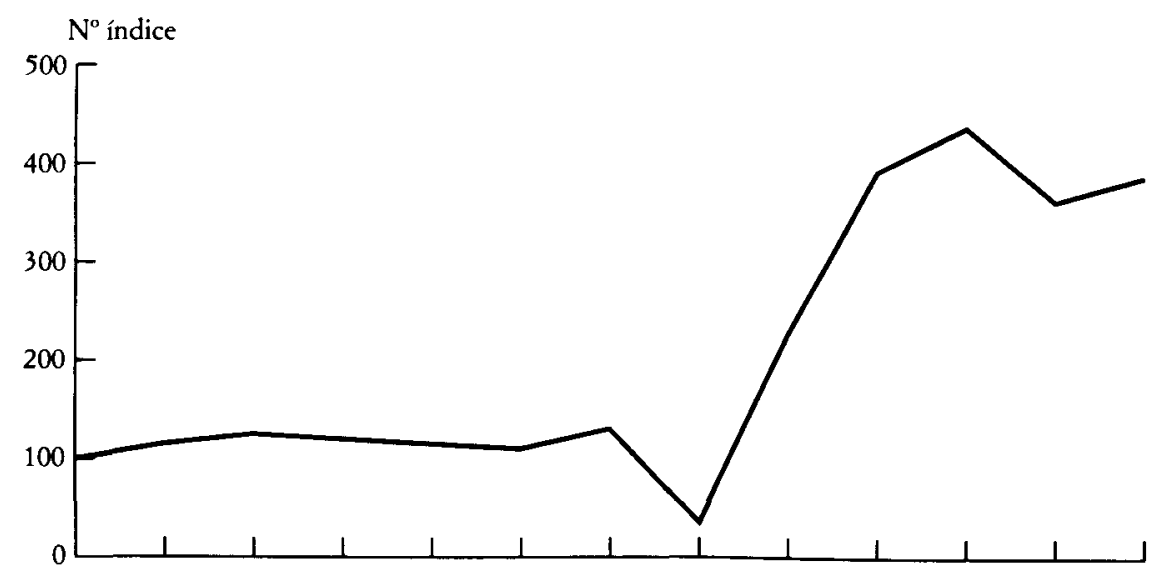

FUENTE: Cuadro 1 (en pesetas constantes).

Básicamente éste sería el marco empírico donde sustentaré mi exposición. Como hemos podido comprobar, la existencia de ciclos de distinta intensidad y duración evidencia la presencia de un mercado de tierras que fluctúa mediatizado por factores claramente coyunturales. Comienzan los primeros años del siglo con unas subidas en los índices muy moderadas (1904: 101,80), como un claro exponente de la bajada del precio de las tierras a consecuencia de la crisis finisecular. A partir de 1905, con un índice del 112.62, indica el inicio de un nuevo ciclo alcista, como lo demuestra el valor alcanzado en 1912, 130,41. Es 
un periodo de fuerte dinamización en las estructuras agrarias de la comarca (sindicatos agrícolas, cajas de ahorros, introducción de nuevos cultivos, proliferación de prospecciones acuiferas, etc.), que propició no solamente una recuperación del valor de la tierra como factor de producción, sino también por la llegada de capitales procedentes de la minería ${ }^{29}$. Es precisamente en este período (1905) cuando se produce la TAV más elevada, un 10,6 por ciento. Hasta 1921 la cotización de las tierras se mantiene alta aunque sea con notorios altibajos, como el de 1917 (119.84), pero también con moderadas subidas, como las de $1913(131,18)$ y $1915(131,44)$. En cualquier caso, los precios corrientes se mantienen al alza hasta 1920 , lo que podría indicar que las fluctuaciones corresponden más bien a una serie de imponderables difíciles de detectar.

Es en la década de los años veinte cuando se produce el descenso general de todos los índices. Hay períodos, como el cuatrienio 1924-1928, que registran los precios más bajos desde 1905. Además el comportamiento de la TAV es claramente negativo como lo demuestra la pérdida de 7,6 puntos en 1922. Pero, ¿cuáles podrían ser las causas de esta depreciación? En una primera aproximación se podría atribuir a las frustradas expectativas que produjo el fracaso de la mayoría de los planes para la construcción de los pantanos Caridad, Taibilla y Fuensanta para el abastecimiento de aguas potables y de riego para Murcia y Cartagena ${ }^{30}$. A pesar de que fue un tema que abarcó todo el primer tercio del siglo $\mathrm{xxv}^{31}$, fue en la década de los veinte cuando se intensificó. En buena lógica, las expectativas de riego aumentarian la presión en el mercado de tierras por el lado de la demanda, incrementando su precio. Probablemente asi fue durante el periodo 1913-1920, manteniendo la subida hasta 1925 , año en el que tanto los representantes del sector agrario, como los del resto de los subsectores económicos que constituyen la estructura productiva del área, comprendieron que el trasvase de caudales hidráulicos a la comarca era un proyecto que, por el momento, había fracasado 32 .

A partir de 1929 comienzan a elevarse los indices (TAV del 9,4 por ciento), volviendo a unos valores muy similares al período 1910-1919. En los años 19301935 el comportamiento es de alza moderada, síntoma de una clara pero lenta recuperación. Durante este quinquenio el comportamiento de los índices refle-

29 Véase mi tesis doctoral Transformaciones Agrarias en la Comarca del Campo de Cartagena, siglos XIX $y$ XX, Universidad de Murcia, 1992.

${ }_{30}$ Mediavilla Sánchez, J. (1925), p. 56.

31 Piñera, Alvaro de la (1990), p. 18.

32 Algo parecido ocurrió en la zona del Bajo Segura a raiz de las esperanzas que en 1923 supuso la posibilidad de dotaciones de aguas por parte de Riegos de Levante. Costa Mas, J. (1981), p. 421. 
ja un repunte de las cotizaciones en 1933, lo que supone un incremento de más de ocho puntos respecto a 1930. Esta tendencia se rompe al año siguiente con una variación interanual de $-4,4$ por ciento. Las causas podrían encontrarse en los efectos que a corto plazo propició la Ley de Reforma Agraria de septiembre de 1932. A pesar de su escasa incidencia en la comarca, el hecho de estar incluidas las principales fincas de la zona en el Registro de la Propiedad Expropiable de Cartagena-Fuente Alamo levantó expectativas compradoras en colectivos de medianos propietarios y labradores acomodados que veían en estas disposiciones una excelente oportunidad de incrementar su patrimonio territorial ${ }^{33}$.

Tras la guerra civil es cuando se producen las subidas más bruscas (TAV 34,7 por ciento) debido al fuerte efecto inflacionista que traerá consigo la posguerra. Pero si dicho contraste pudiera parecer evidente, no lo es tanto las fluctuaciones anuales que experimentan los precios en el periodo 1940-1950. La política agrícola del período franquista daba prioridad a los cereales, convirtiéndolos en el producto leader de la producción agricola española. Como apuntó Carlos Barciela: «La posición era elemental y clara: disponer de suficientes cantidades de grano para atender las necesidades de alimentación de la población» ${ }^{34}$.

Es más, la evolución de su precio servía de referencia para el resto de los cultivos. Lo cierto es que a partir de este año se produjo un exceso de la demanda hacia unas tierras cuyo valor de cambio habia estado tradicionalmente muy por debajo del resto de los productos agrícolas. Pero además las inversiones en tierras se llevaron a efecto por la ventajosa relación entre precio y rentabilidad agraria. Como ha señalado Sumpsi «De este modo se comprende que la acumulación de capital de la burguesía agraria se destinara, prioritariamente, a modernizar la explotación y a comprar nuevas fincas, en lugar de canalizarse hacia inversiones industriales o de equipamiento» ${ }^{35}$.

Asi es; durante la décadas de los años cuarenta se alcanzan los indices más elevados de la serie, siendo especialmente significativo los años 1948-1950, este último con una subida del 481,95. Las variaciones interanuales no registran ningún signo negativo en contraste con el resto de los ciclos analizados. Al comienzo de la década de los cincuenta, y a pesar de que no hubieron grandes cambios a nivel de estructuras agrarias, comienzan a descender los índices, aunque nunca a los niveles de los años cuarenta. Tal como indican las tasas interanuales, éstas caen en casi todos los años aunque a un ritmo muy modera-

33 Román Cervantes, C (1991c), pp. 43-59.

34 Garcia González, A., y Barciela López, C. (1986), p. 504.

35 Sumpsi, J. M." (1986), p. 45. 
do. Las causas quizá se deban buscar en una serie de hechos: 1. Estabilización del mercado de tierras debido a la política de préstamos hipotecarios especiales emprendida en estos años. 2. Aumento de la tasa de liquidez monetaria de los agricultores y, en consecuencia, posibilidad de dirigir sus inversiones hacia cultivos de más elevada rentabilidad, y 3. Como resultado de lo anteriormente expuesto, las tierras empleadas en secano-cereal experimentaron una fuerte depreciación ${ }^{36}$. También pudo deberse, pasados los años de la posguerra, a un exceso de oferta tras el periodo de «faim de terres» de los años cuarenta. En cualquier caso, los problemas en los sistemas de aprovisionamiento alimenticio en el país se estaban regularizando y una nueva orientación en materia de política agrícola se estaba imponiendo, buscando en última instancia la modernización de la agricultura ${ }^{37}$.

De lo expuesto sobre el comentario del cuadro pueden hacerse dos consideraciones. En primer lugar, que la elección de las tierras de secano-cereal para cuantificar la evolución de su precio ha sido acertada por tratarse de un cultivo durante la mayoría de los años de estudio de demanda inelástica, por lo que sus variaciones no son en cualquier caso tan fuertes como las de otros cultivos más dependientes con las fluctuaciones del mercado. Por ello, y a pesar de las distintas intensidades ciclicas anteriormente descritas, considero que se trata de un mercado de tierras estable y uniforme. En segundo lugar, los precios que he obtenido en pesetas corrientes son precios reales, como pude comprobar al cotejarlos con otros de áreas agrícolas cercanas (en los secanos de la vega del Bajo Segura el precio por hectárea para 1960 era de 4.637 ptas.) ${ }^{38}$. Por todo ello estimo que la serie puede considerarse como válida a la espera de otros trabajos que se proyecten sobre el resto de las comarcas de la Región de Murcia.

Como anuncié al principio, quisiera proceder a exponer los valores del resto de los cultivos que tradicionalmente han formado parte del output agricola de la comarca: olivo, higuera, almendro, algarrobo, viña y huerta. Me aproximaré a sus precios unitarios a través de un variado material heurístico obligado por la dificultad de localizar series más homogéneas. Puesto que las diferencias cronológicas de la serie son evidentes, no está en mi ánimo realizar un análisis preciso de la evolución del precio de la tierra en un periodo tan dilatado, sino más bien comentar los valores que los distintos aprovechamientos han alcanzado en el año del sondeo y, en consecuencia, mostrar qué cultivos eran los que experimentaban las oscilaciones más intensas.

\footnotetext{
36 Román Cervantes, C. (1991), mimeo.

37 Barciela López, C. (1986), p. 419.

38 Costa Mas, J. (1981), p. 422.
} 


\section{CUADRO 2}

Valores medios unitarios de la tierra por aprovechamientos. Campo de Cartagena. Años 1866-1974 (pesetas constantes, año $1900-100$ ) (1)

\begin{tabular}{|c|c|c|c|c|c|c|}
\hline \multicolumn{7}{|c|}{ Cultivos (ptas./has.) } \\
\hline Años & Olivo & Higuera & Almendro & Algarrobo & Viña & Huerta \\
\hline 1866 & 403 & - & - & - & 114 & 1.161 \\
\hline $1886 \ldots \ldots \ldots \ldots$ & 1.013 & - & - & - & 1.317 & 1.519 \\
\hline $1900 \ldots \ldots \ldots \ldots$ & 989 & 721 & 1.000 & - & 831 & 4.377 \\
\hline 1905 & 845 & 856 & 1.145 & - & 835 & 4.517 \\
\hline 1910 & 756 & 674 & 1.132 & 1.290 & 760 & 4.787 \\
\hline$\ldots \ldots \ldots$ & 641 & 743 & 1.355 & 817 & 1.030 & 4.336 \\
\hline 1930 & 965 & 1.020 & 1.961 & 944 & 1.534 & 4.194 \\
\hline 1940 & 1.734 & 1.523 & 3.541 & 2.491 & 2.732 & 7.180 \\
\hline $1965 \ldots \ldots \ldots \ldots$ & 2.138 & 3.434 & 3.434 & 3.434 & 4.759 & 6.525 \\
\hline $1974(2) \ldots \ldots \ldots \ldots$ & 10.479 & 10.041 & 14.513 & 8.033 & 10.041 & 61.616 \\
\hline
\end{tabular}

(1) El indice deflactor de los años 1866,1886 y 1900 se ha calculado con el indice general de precios obtenido a partir de las estimaciones de María del Carmen Arenales (1974), p. 1121 Para el resto del período, Alcaide, A (1974), pp. 1142-1143.

(2) Los precios de 1974 reflejan en cierto modo una actualización por parte de la Administración Central ante las nuevas perspectivas que se abrian para la comarca con la llegada de las aguas del Trasvase Tajo-Segura.

FuENTE: 1866: Promedios obtenidos a partir del A.C.F.C, Interrogatorio de 1866. La superficie ha sido pasada a hectáreas y la unidad de cuenta a pesetas; 1886: Datos procedentes de la Dirección General de los Registros Civil de la Propiedad y del Notariado. Valor aproximado de una bectárea de terreno a 31 de diciembre de 1886 con distinción según la especie de cultivo. Memorias y Estados formados por los registradores de la propiedad en cumplimiento de lo prevenido en el R. D al 31 agosto de 1886, Madrid, 1890, Vol. IV, p. 87; 1900-1940: A.P.N.C, Años 1900-1940. Elaboración propia a partir de las escrituras de compraventa; 1965: A.C.A.C. Relación de valores unitarios de la tierra y su distribución en el término comparados con los que se proponen por el Servicio Provincial del Catastro para el año próximo, Cartagena, 19 de junio de 1965. Promedios entre las distintas calidades de tierras; 1974: Valores obtenidos de los precios máximos y mínimos, publicados en el Plan General de transformación de las Zonas Regables del Campo de Cartagena, Organización Sindical de Murcia y Hermandades de Labradores y Ganaderos del Campo de Cartagena, junio de 1974, Cartagena, p. 15. Elaboración propia.

Creo que no es necesario hacer referencia a los precios de las tierras con cereales, ya que éstas solian tener unas cotizaciones más bajas, como pudo comprobarse en los datos del Cuadro 1 . Los de las tierras con olivo indican un crecimiento durante la segunda mitad del siglo XIX, alcanzando las 1.013 ptas./has. en 1886. A comienzos de la actual centuria comienzan a descender, caída que comprende casi todo el primer tercio del siglo $\mathrm{xx}$ mientras se apreciaban el resto de las cotizaciones del arbolado de secano. En 1940 y en 1965 se rompe la tendencia aumentando su valor, pero siempre por debajo del resto de los árboles. La crisis 
que la economía oleícola arrastró desde las últimas décadas del siglo XIX y primer tercio de la actual centuria incidió negativamente en los precios de las tierras que contenían olivares ${ }^{39}$. Además, la escasa productividad de las variedades con mayor difusión en la zona (manzanilla y cornicabra) supuso una detracción de la demanda de suelo agrario dedicado a su cultivo. Un comportamiento similar aunque con unos precios más sostenidos es el de la higuera, que pasa de 721 ptas./has. en 1900 a 1.523 ptas./has. para 1940. Es decir, su precio se ha incrementado en cuarenta años un 52,65 por ciento, superando al olivo en 1920 y en 1930. La cifra de 1965 es el resultado de una estimación que afecta por igual al almendro y al algarrobo, ya que la documentación los incluía en el mismo apartado de los frutales.

En cuanto a las tierras cultivadas con almendros, su alta rentabilidad, sobre todo durante el primer tercio del siglo, hizo que obtuviera las cotizaciones más elevadas. Sin datos de 1866, ni tampoco de 1886, sale al mercado en 1900 con un valor por encima del resto de los árboles (olivo e higuera). La subida más espectacular tiene lugar entre las décadas 1920 y 1940, que pasa de 1.355 ptas./ has. a 3.541 ptas./has., lo que supuso un incremento del 61,73 por ciento. Debo señalar que es precisamente en el periodo $1922-1930$ (ver Cuadro 1) cuando las tierras con cereal tocan fondo a consecuencia de la expansión que está experimentando el almendro como cultivo rentable. Tendencia que se mantiene hasta los años setenta, cuya valoración unitaria, 14.513 ptas. es la más elevada después de las tierras de regadío. Los suelos que contenían algarrobos tuvieron en ocasiones - 1910, 1920 y 1940 - unas cotizaciones por encima del olivo y la higuera, pero nunca alcanzó las del almendro. Los primeros datos proceden de 1910 con un precio unitario muy elevado, 1.290 ptas. como corresponde a un cultivo nuevo, ya que al comenzar las plantaciones en 1900 , es precisamente durante la segunda década del presente siglo cuando los árboles se encontraban en plena producción. Además, hay que tener en cuenta que su cultivo no estaba muy extendido, lo cual podría ser un elemento que incidiera positivamente en su valoración. Para el último año 1974 , puede observarse que pierde posiciones $(8.033$ ptas./has.) con respecto a las cotizaciones del resto del arbolado.

Las tierras dedicadas al cultivo de la vid tienen unos valores que van a estar durante todo el periodo por encima de los frutales de secano. Por más que revisé los datos de 1866 éstos apuntaban a una media de 114 ptas./has., por debajo inclusive de los suelos dedicados a cereal. Pienso que la cifra puede estar sesgada a la baja, por lo que no me detendré más en su análisis. Por el contrario, el informe de la Dirección General de los Registros de 1886, sí sitúa en su lugár la cotización alcanzando las 1.317 ptas. Como es sabido, los efectos de la filoxera en Francia

39 Zambrana Pineda, J. F. (1985), pp. 301-320. 
iban a propiciar un aumento en la exportación de los caldos españoles, significando a la postre una apreciación de los de suelos vitivinícolas. A partir de 1900 se empiezan a notar los efectos que la plaga tuvo en la comarca tras su invasión en 1894, provocando una oferta masiva de parcelas con viña y, por consiguiente, induciendo a la caída de las cotizaciones. Puede observarse cómo el valor más bajo se alcanza precisamente en 1900 , con 831 ptas./has., para ir recuperándose paulatinamente durante las décadas siguientes. En 1930 superá con diferencia a los demás cultivos, menos al almendro, tendencia que continúa en el resto de los años analizados. Por último, las tierras dedicadas a huerta y demás cultivos intensivos son, como era de esperar, las que alcanzan los valores más elevados. En efecto, si se toman como referencia los precios de las tierras de almendro, las diferencias son lo suficientemente elocuentes, $1900: 77,15$ por ciento, 1905: 74,65 por ciento, 1910: 76,35 por ciento, 1920: 68,75 por ciento, 1930: 53,24 por ciento, $1940: 50,68$ por ciento, $1965: 63,81$ por ciento y $1974: 47,37$ por ciento. Si proyectáramos estos cálculos hacia las tierras de secano, el contraste sería mayor.

Con el ánimo de precisar aún más las cotizaciones de las tierras por aprovechamientos y comprobar cómo éstas fluctúan dependiendo de la calidad de las mismas, he confeccionado el siguiente cuadro a partir de los datos de la Cámara Agrícola de Cartagena. En él muestro una síntesis de los cultivos más representativos, relacionándolos con aquellos que han sido objeto de análisis a lo largo del presente trabajo. Sin entrar en las diferencias porcentuales entre las categorías de suelos, sí debo insistir en lo que respecta a secano y regadío. De todos es el olivo el que acumula las diferencias más acusadas, pasando de 5.364 ptas./has. secano de $1 .^{a}$ a 37.500 ptas./has. para las de regadío. Estas experimentan una sobrevaloración de un 85,69 por ciento, porcentaje que se incrementa aún más en los suelos de $2{ }^{\text {a }}$, pasando al $90,85 \%$. Llaman la atención estos valores, ya que en el resto de los cultivos, algunos supuestamente más rentables, no alcanzan estas diferencias. Por ejemplo, los frutales están entre el 59,37 por ciento para las de $1 .^{\mathrm{a}}$ y el 49,02 por ciento en las de $3{ }^{\mathrm{a}}$, la viña entre el 56,16 por ciento las de $1 .^{\mathrm{a}} \mathrm{y}$ el 46,54 por ciento las de $3{ }^{\text {a }}$, etc.

Los precios indican, según sean las calidades de los suelos, que hay fluctuaciones más intensas en los cultivos menos rentables que en aquellos que poseen una mayor cotización. Asi es; en el olivo de secano los precios caen bruscamente llegando a descender hasta un 61,01 por ciento para las tierras de $2 .^{\mathrm{a}}$ y un 52,17 por ciento en las de 3.a. También en el regadio se mantiene el mismo comportamiento aunque la bajada es mas moderada, un 39 por ciento en los suelos de $2 .^{\mathrm{a}} \mathrm{y}$ un 62,24 por ciento en los de 3.a. En buena lógica, y en la medida que se incrementa la rentabilidad del cultivo, las diferencias porcentuales entre las calidades de los suelos disminuyen. Así ocurre para los frutales de secano, el 19,76 por cien- 
to para las de $2 .^{a}$ y el $36,59 \%$ para las de $3 .^{\mathrm{a}}$, y en el regadio, un 21,31 por ciento y un 48,48 por ciento respectivamente. Pero si puede parecer significativo el contraste con respecto al olivo, éste se acentúa notoriamente cuando paso a analizar las cifras referidas a la vid. Tanto en secano como en regadío abarca los porcentajes más pequeños entre las distintas calidades de suelos: un 16,13 por ciento y el 19,22 por ciento para las tierras en secano de $2 .^{\text {a }}$ y $3 .^{\text {a }}$ y el 33,33 por ciento y 16,66 por ciento para las de regadío. En cuanto a las tierras con cítricos son las que tienen una mayor cotización, 74.454 ptas, precio que puede considerarse bajo si se compara con los que alcanzarán en la década de los setenta, llegándose a pagar la hectárea en pesetas corrientes entre 225.000 y 450.000 ptas ${ }^{40}$.

\section{CUADRO 3}

Valor unitario por cultivos y calidad de las tierras en el término municipal de Cartagena. Año 1965 (pesetas corrientes)

\begin{tabular}{|c|c|c|c|}
\hline Cultivo (1) & Calidad & Secano & Regadio (2) \\
\hline Olivar & $1 .^{\mathrm{a}}$ & 5.364 & 37.500 \\
\hline Olivar & 2. & 2.091 & 22.875 \\
\hline Olivar ..... & $3 .^{a}$ & 1.000 & 8.636 \\
\hline Frutales (3) & 1. & 9.292 & 22.875 \\
\hline Frutales (3) .. & 2. & 7.455 & 18.000 \\
\hline Frutales (3) .. & $3 .^{a}$ & 4.727 & 9.273 \\
\hline Viña ....... & $10^{a}$ & 19.727 & 45.000 \\
\hline Viña $\ldots$. & 2. & 16.545 & 30.000 \\
\hline Viña .... & 3. & 13.364 & 25.000 \\
\hline Huerta .... & 1. & - & 30.870 \\
\hline Huerta ...... & 2. & - & 23.478 \\
\hline Huerta $\ldots \ldots$ & 3. & - & 16.085 \\
\hline Citricos (4) & $1 .^{a}$ & - & 74.454 \\
\hline Citricos (4) ... & 2, & - & 55.636 \\
\hline Citricos (4) ... & $3 .^{2}$ & - & 36.818 \\
\hline Pastizales (5) & 1. & 135 & - \\
\hline Pastizales (5) $\ldots \ldots \ldots \ldots$ & 2. & 105 & - \\
\hline Pastizales (5) $\ldots \ldots \ldots$ & $3 .^{a}$ & 75 & - \\
\hline
\end{tabular}

(1) He agrupado los cultivos más representativos de la clasificación.

(2) Los datos de regadio hacen referencia a riego con agua propia, ya que las conto.

la tierras con agua comprada tienen una valoración a la baja entre un

(3) En los frutales se incluyen almendros, higueras y algarrobos.

(4) Datos correspondientes a naranjales.
(5) Debo señalar la distinción entre «pastizal» y «pastizal del Estado». En ambos casos po. seen los mismos precios.

FUENTE: A.C.A.C.

40) Costa Mas, J. (1981), p. 422. 


\section{TENDENCIAS ACTUALES}

Para terminar quisiera mostrar cómo está en la actualidad la cotización de tierras en la Región de Murcia. No voy a proceder a un análisis exhaustivo de la información, ya que las cifras no están desagregadas por comarcas, lo que impide cotejar los resultados entre las distintas áreas agricolas de la Región. Excepto los cereales, no puede tener la misma valoración, por poner tan sólo un ejemplo, una hectárea de viña en la Comarca del Altiplano (Yecla y Jumilla) que en el Campo de Cartagena, al igual que otros árboles frutales tanto de secano como de regadío. Por ello, es mi intención mostrar cuáles son los resultados que se están obteniendo actualmente en la Sección de Estadistica de la Consejería de Agricultura respecto a las fluctuaciones en los precios de las tierras. La serie histórica comprende de 1981 a 1990, computandose unos precios máximos y minimos, obteniendo la media ponderada y, en consecuencia, la cotización más frecuente.

\section{CUADRO 4}

Media ponderada regional de los precios más frecuentes por aprovechamientos y cultivos de secano. Años 1981-1990 (en miles de pesetas, año 1981 - 100)

\begin{tabular}{|c|c|c|c|c|c|c|c|c|c|}
\hline & \multirow{2}{*}{ Años } & \multicolumn{2}{|c|}{ Labor } & \multicolumn{2}{|c|}{ Frutos secos } & \multicolumn{2}{|c|}{ Viña } & \multicolumn{2}{|c|}{ Pastizal } \\
\hline & & $I$ & II & $I$ & II & $I$ & II & $I$ & II \\
\hline 1981 & $\ldots$. & 175 & 175 & 300 & 300 & 285 & 285 & 30 & 30 \\
\hline 1982 & & 185 & 162 & 290 & 255 & 285 & 250 & 30 & 26 \\
\hline 1983 & & 220 & 173 & 375 & 294 & 260 & 204 & 35 & 27 \\
\hline 1984 & $\ldots$ & 225 & 158 & 385 & 271 & 260 & 183 & 35 & 25 \\
\hline 1985 & & 300 & 196 & 400 & 261 & 250 & 163 & 45 & 29 \\
\hline 1986 & & 315 & 185 & 490 & 288 & 235 & 138 & 45 & 26 \\
\hline 1987 & $\ldots$ & 300 & 167 & 525 & 290 & 330 & 183 & 55 & 31 \\
\hline 1988 & & 425 & 223 & 665 & 349 & 330 & 173 & 45 & 24 \\
\hline 1989 & & 400 & 196 & 620 & 305 & 375 & 184 & 44 & 22 \\
\hline 1990 & $\ldots \ldots \ldots \ldots \ldots \ldots$ & 270 & 124 & 445 & 204 & 420 & 192 & 45 & 21 \\
\hline
\end{tabular}

I. Pesetas corrientes; II. Pesetas constantes. Indice deflactor obtenido a partir PIB (a precios de mercado), I.N.E (1992): Contabilidad Nacional de España, serie enlazada 1964-1991, Madrid, p. 41. Fuente: Consejeria de Agricultura, Ganadería y Pesca.

A lo largo de los últimos diez años las tierras de labor-secano están experimentando una baja por exceso de oferta, mientras que la demanda es prácticamente inexistente. A precios de 1981 la tendencia es irregular, destacando el pe- 
ríodo 1985-1989, donde se registran las subidas más significativas. Por el contrario, los de 1990 indican en términos relativos que se ha producido una depreciación de un 41,12 por ciento en el transcurso de una década. Las causas parecen encontrarse en una bajada en los precios de los cereales frente a los incrementos de los costos de producción.

\section{GRAFICO 2}

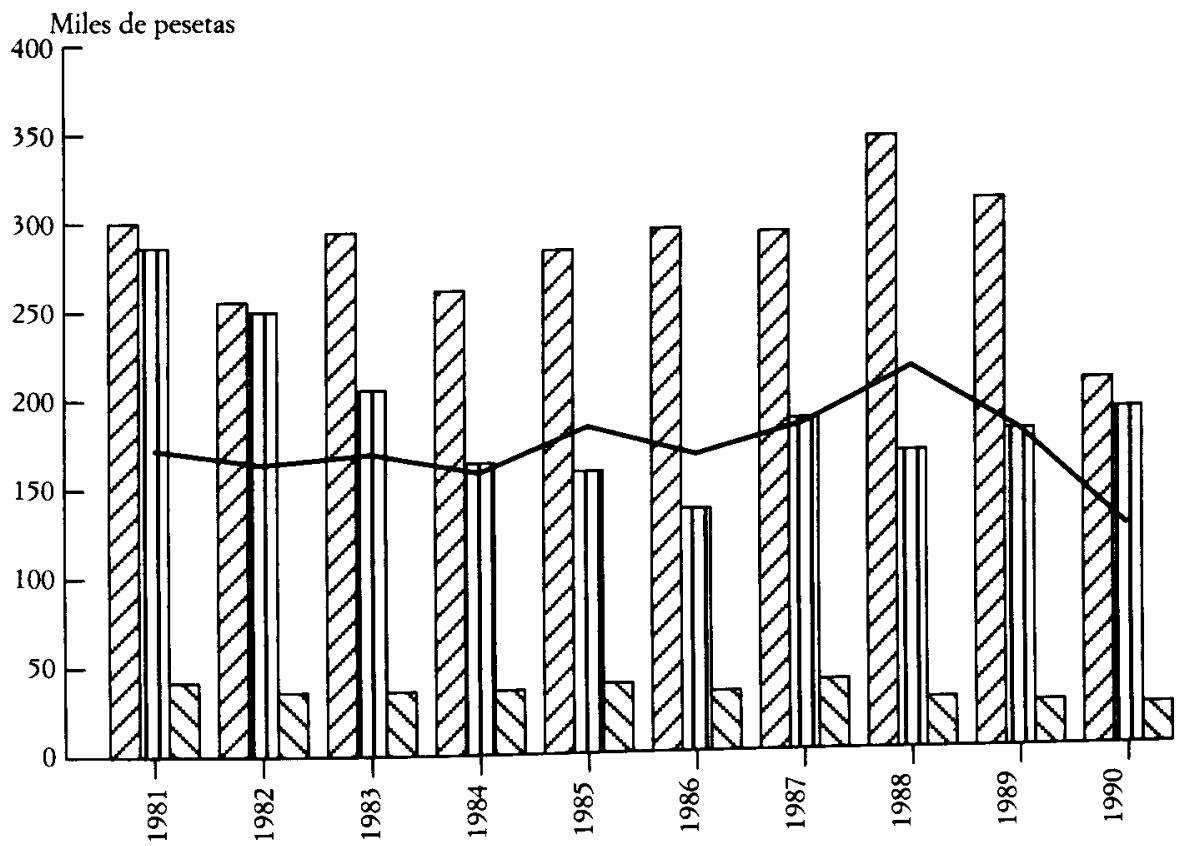

Fuentr: Cuadro 4 (en pesetas constantes).

Pero además hay un elemento de carácter coyuntural ocasionado por las continuas elevaciones de los tipos de interés del dinero que van a incrementar los rendimientos del capital. Otros motivos podrían ser la revisión de las tasas impositivas que gravan la tierra, las malas cosechas, etc. Aunque algunas de estas causas pueden hacerse imputables también al resto de los cultivos, es al grupo de los cereales, por su baja rentabilidad, a quien más afecta. En cuanto a los frutos secos (almendra), tiene actualmente un comportamiento bajista, al pasar de 349.000 ptas en 1988 a 204.000 ptas en 1990 , lo que supone un des- 
censo del 41.54 por ciento. Estas depreciaciones se producen a pesar de una serie de circunstancias proclives a incrementar el cultivo de los frutos secos de secano y más concretamente del almendro. Entre ellos, citar la ayuda aprobada por la Comunidad Económica Europea a la «mejora de la calidad y producción» de las explotaciones, fijadas en 45.809 ptas./has. para los primeros años y en 32.108 ptas./ha. para los 5 años siguientes. En contraste con las cotizaciones en los últimos años de los cereales y frutos secos, son las de viña de secano las que experimentan una apreciación,, pasando de 184.000 ptas./has. en 1989 a 192.000 ptas./has. en 1990, un incremento en términos relativos del 4,16 por ciento. Los motivos no deben encontrarse en las cotizaciones del vino en los mercados de productos alimenticios, sino más bien actúan como variable inflacionista las primas concedidas por la administración para la reducción y abandono de la superficie dedicada a la vid.

\section{CUADRO 5}

Media ponderada regional de los precios más frecuentes por aprovechamientos y cultivos de regadío. Años 1981-1990 (en miles de pesetas/año 1981 - 100)

\begin{tabular}{|c|c|c|c|c|c|c|c|c|c|}
\hline & \multirow{2}{*}{ Años } & \multicolumn{2}{|c|}{ Labor } & \multicolumn{2}{|c|}{ Frutos secos } & \multicolumn{2}{|c|}{ Viña de mesa } & \multicolumn{2}{|c|}{ Limón } \\
\hline & & $I$ & $I I$ & $I$ & II & $I$ & $I I$ & $I$ & II \\
\hline 1981 & & 1.020 & 1.020 & 1.430 & 1.430 & 1.095 & 1.095 & 3.085 & 3.085 \\
\hline 1982 & & 1.030 & 904 & 1.420 & 1.247 & 1.080 & 948 & 3.035 & 2.665 \\
\hline 1983 & & 1.040 & 816 & 1.400 & 1.099 & 1.100 & 863 & 3.645 & 2.861 \\
\hline 1984 & & 1.060 & 745 & 1.405 & 988 & 1.130 & 795 & 3.405 & 2.395 \\
\hline 1985 & & 1.040 & 679 & 1.475 & 963 & 1.165 & 761 & 3.400 & 2.221 \\
\hline 1986 & & 1.330 & 782 & 1.390 & 817 & 1.520 & 894 & 3.215 & 1.890 \\
\hline 1987 & & 1.500 & 833 & 1.165 & 647 & 1.830 & 1.016 & 3.200 & 1.777 \\
\hline 1988 & & 1.900 & 998 & 1.100 & 578 & 2.000 & 1.051 & 3.200 & 1.682 \\
\hline 1989 & & 1.965 & 965 & 1.170 & 575 & 2.230 & 1.095 & 3.475 & 1.707 \\
\hline 1990 & & 1.900 & 870 & 975 & 446 & 2.050 & 939 & 3.175 & 1.454 \\
\hline
\end{tabular}

I. Pesetas corrientes; II. Pesetas constantes. Indice deflactor obtenido a partir del PIB (a precios de mercado), I.N.E (1992): Contabilidad Nacional de España, serie enlazada 1964-1991, Madrid, p. 41.

Fuente: Consejería de Agricultura, Ganadería y Pesca.

En cuanto a los cultivos de labor-regadio, también experimentan, al igual que sus homónimos de secano, un comportamiento irregular aunque predomina el descenso de las cotizaciones. En términos relativos y en pesetas de 1981, 
las tierras de regadio dedicadas al sistema cereal han experimentado un descenso del 17,24 por ciento. Las causas podrían estar, según la Sección de Estadistica, en las malas campañas de la alcachofa, del pimiento pimentonero y del algodón. Por el contrario, apunta como factores estabilizadores los siguientes: «De todas formas la ubicación del regadio en la Vega Media del Segura, Campo de Cartagena y Valle del Guadalentín con agricultores de gran experiencia, buena infraestructura, parcelas de poca dimensión, buena climatología y agua, frena la oferta y evita una caída mayor de los precios» ${ }^{41}$. El subrayado quiere insistir en el grado de eficacia que aún hoy día conservan las unidades de explotación relativamente pequeñas propias de la agricultura familiar, en contra de aquellas opiniones partidarias de grandes concentraciones territoriales, de la presencia de grandes empresas con capital multinacional y, en fin, de una monopolización de la agricultura. Una vez más la pequeña y mediana propiedad

\section{GRAFICO 3}

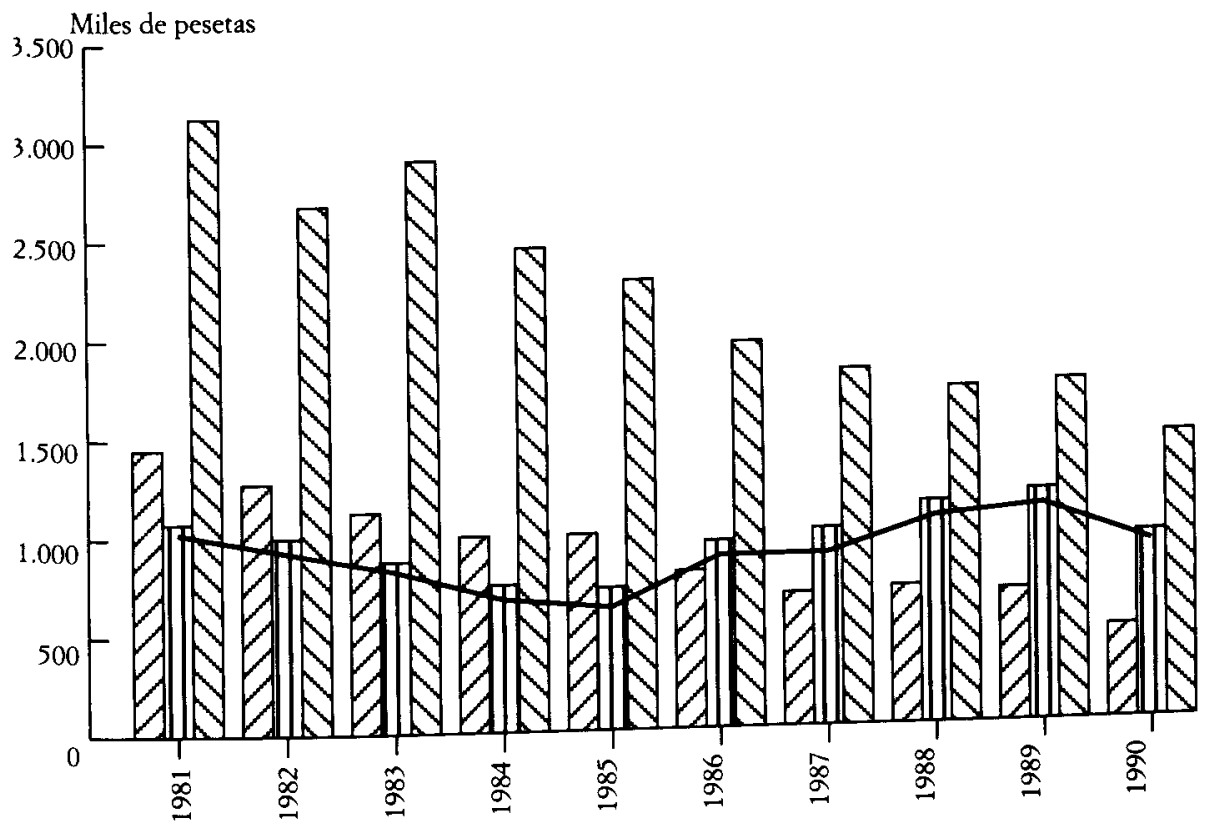

Fuente: Cuadro 5 (en pesetas constantes).

4 Informe, Sección de Estadística, 1991, Consejería de Agricultura, Pesca y Alimentación 
sirve de reguladora de las estructuras agrarias y más concretamente de los mercados locales de tierras ${ }^{42}$.

En lo concerniente al resto de los cultivos, quisiera reseñar tan sólo la cotización del limón en 1990 ( 1.454 .000 ptas.), que va perdiendo posiciones progresivamente desde su valor más elevado en 1981. El comentario que la Sección de Estadística de la Consejería de Agricultura realiza sobre las cotizaciones de este cultivo lleva a pensar, aun estando pendientes de estudios más precisos, que el precio unitario del suelo en la Comarca del Campo de Cartagena ha sido y es uno de los más elevados de la región: «En los precios del año 90 se produce una baja sensible debido a que en años anteriores se producía una sobrevaloración en el Campo de Cartagena debido a causas diversas, precios que han evolucionado a la baja y se han equiparado a los de la Vega del Segura y Valle del Guadalentín» ${ }^{43}$.

\section{RECAPITULACION}

A lo largo de las líneas precedentes he pretendido aproximarme a la evolución del precio de la tierra aplicado a un modelo local. Pudimos comprobar la estrecha dependencia entre renta y precio, conexión que está actualmente vigente y que regula las cotizaciones de las tierras, a pesar de la fuerte presión que reciben aquellas que poseen unas excelentes rentas de localización: proximidad a zonas turísticas y cercanía a polígonos de desarrollo industrial y urbano de algunas de las localidades que forman parte de la comarca (Cartagena, Torre Pacheco, San Javier y San Pedro del Pinatar). Estas son susceptibles de recibir una fuerte demanda por parte de grupos financieros y empresariales, muy capitalizados y con intereses en la zona.

Los valores obtenidos en las tierras dedicadas al sistema cereal indican la estrecha relación con el precio de los cultivos, donde el aumento de la demanda se convierte en la variable de mayor impacto en la oscilación de las cotizaciones. Prueba de ello son las bruscas subidas del periodo de la posguerra, propiciadas no solamente por la importancia de los cereales debido a la política agraria del primer franquismo, sino porque la tierra se convirtió en un valor

42 Román Cervantes, C. (1993) (en prensa).

43 Informe, Sección de Estadística, 1991. Entre las «causas diversas», podrían encontrarse la proximidad a las zonas turisticas que ejercen una fuerte presión por el lado de la demanda sobre unas tierras recientemente regadas por el Trasvase Tajo-Segura. A ello habria que añadir la mejora de la infraestructura viaria, asi como las continuas inversiones en equipamientos que realizan los empresarios agrícolas. 
refugio donde se dirigieron abundantes capitales locales. En cuanto a las tierras dedicadas al resto de los cultivos de secano, han tenido unas valoraciones por encima de las de los cereales, sobre todo durante el primer tercio del siglo $\mathrm{xx}$, que es cuando la difusión de las plantas arbóreas alcanzan importantes extensiones de superficie agrícola. De entre ellas, las dedicadas al cultivo del almendro y del algarrobo, que experimentaron alzas en unos años donde la demanda procedente de los mercados internacionales de frutos secos iba en aumento.

La inclusión de las tendencias actuales se explica por entender cuál fue la trayectoria histórica de los aprovechamientos más tradicionales y cuál es su grado de cotización. Se ha comprobado por poner tan sólo un ejemplo, cómo las tierras vitivinicolas secularmente con valoraciones por encima de algunos ärboles de secano, se encuentran infravaloradas a pesar del repunte que están experimentando actualmente motivado por la politica de subvenciones para su abandono. Las actuales normativas comunitarias en materia de política agrícola están obligando a encontrar nuevos sistemas de planificación y ordenación de los cultivos y buscar el equilibrio en los mercados de los productos agrarios. En definitiva, se produce una depreciación del suelo agrario tanto en los secanos como en los regadios, lo que demuestra de qué modo el uso de la tierra como factor de producción a la agricultura, ha perdido peso específico en los mercados territoriales frente a criterios valorativos basados en la localización del terrazgo.

\section{BIBLIOGRAFIA}

AlCAIDE, Angel (1976): «Una revisión urgente de las series de renta nacional española en el siglo XX», Datos básicos para la Historia Financiera de España 1850-1975, Vol., I, Madrid, Instituto de Estudios Fiscales, Ministerio de Economía y Hacienda, pp. 1127-1150.

Arenales, María del Carmen (1976): «Un indicador de precios de la economia española para el periodo 1850-1900», Datos básicos para la Historia Financiera de España 1850-1975, Vol., I, Madrid, Instituto de Estudios Fiscales, Ministerio de Economía y Hacienda, pp. 1111-1123.

Arnalte Alegre, Eladio, y Avella Reus, Lorenzo (1986): «Modelos locales del mercado de la tierra en el País Valenciano", Agricultura y Sociedad, n. ${ }^{\circ} 41$, Madrid, MAPA, pp. 139-183.

Barciela Lopez, Carlos (1986): «Introducción», Historia Agraria de la España Contemporánea, Vol. 3, Barcelona, Crítica, pp. 383-454.

Bornon, Jean-Pierre y Cavailhés, Jean (1988): «Essai d'explication de la baisse du prix des terres», Etudes Rurales, n. ${ }^{\circ}$ 110-111-112, avr-déc, Paris, pp. 215-236. 
Costa Mas, José (1981): «Cambios de las estructuras agrarias al Sur de Alicante (aproximación al mercado de la tierra en el Bajo Segura)», La propiedad de la tierra en España, Alicante, Universidad de Alicante, pp. 419-436.

Cruz Villalon, Josefina (1981): «Transformaciones recientes de la estructura de la propiedad en el Valle del Bajo Guadalquivir», La propiedad de la tierra en España, Alicante, Universidad de Alicante, pp. 241-252.

Domingo, Teresa et al. (1987): Métodos y ejercicios de economía aplicada, Madrid, Pirámide.

Garcia González, Aurelio, y Barciela Lopez, Carlos (1986): «Un análisis crítico de las series estadísticas de los precios del trigo entre 1937-1980», Historia Agraria de la España Contemporánea, Vol. 3, Barcelona, Crítica, pp. 508-509.

GONZALEZ, Manuel Jesús (1990): «La autarquía económica bajo el régimen del general Franco: una visión desde la teoría de los derechos de la propiedad», Información Comercial Española, diciembre-enero, Madrid, Ministerio de Economia y Hacienda, pp. 19-31.

Mediavilla Sánchez, José (1925): Memoria, sobre la construcción de los pantanos de la Caridad, Taibilla y Fuensanta, para aprovechamiento de aguas potables y de riegos para Murcia y Cartagena, Cartagena.

Molinero Hernando, Luis (1981): «Contribución al estudio de las transformaciones recientes de las explotaciones en Castilla La Vieja», La Propiedad de la tierra en España, Alicante, Universidad de Alicante, pp. 253-265.

NAREDO, José Manuel (1989): «Precio y Renta de la tierra», Actas del IV Congreso Nacional de la Asociación de Historia Económica, Alicante, Universidad de Alicante: mimeo.

Parias SAINZ DE Rozas, María (1989): El mercado de la tierra Sevillana en el siglo XIX, Sevilla, Diputación Provincial y Universidad de Sevilla.

PIÑERA, Alvaro de la (1990): «Breve historia del abastecimiento de aguas de Cartagena», Cuadernos del Estero, n. ${ }^{\circ}$ 4, julio-diciembre, Cartagena, pp. 5-26.

ROBLEDO, Ricardo (1983): «Notas sobre el precio de la tierra en España (1836-1914)», Revista de Historia Económica, Año I, n. ${ }^{\circ}$ 2, Madrid, pp. 253-275.

Roman Cervantes, Cándido (1991 a): «Respuesta a la crisis: desarrollo de los modelos de financiación agraria en el Campo de Cartagena (1880-1930)», Estudis d'Historia Económica, n. ${ }^{\circ}$ 2, Palma de Mallorca, pp. 111-128.

(1991 b): «La modernización de las explotaciones agrarias a través del crédito oficial: el ejemplo del Banco Hipotecario de España», Encuentros de Historia Económica, Universidad Internacional Menéndez y Pelayo, Valencia: mimeo.

- (1991 c): «Alcance y significado de la Reforma Agraria de 1933 en la Comarca del Campo de Cartagena», Cuadernos del Estero, n. ${ }^{\circ}$ 5, enero-junio, Cartagena, pp. 43-59.

(1993): «La propiedad de la tierra en la Comarca del Campo de Cartagena, siglos XIX y XX», Areas, n. ${ }^{\circ} 15$, Murcia (en prensa).

Sánchez Rodriguez, Porfirio (1986): «La encuesta de precios de la tierra del Ministerio de Agricultura, Pesca y Alimentación», Agricultura y Sociedad, n. ${ }^{\circ} 41$, Madrid, MAPA, pp. 187-207.

SClARANDIS, Clara (1987): «Structura della propietá e mercato della terra in una comunità piemontese del XVIII secolo», Quaderni Storici, n. ${ }^{\circ} 65$, año XXII, agosto, Urbino, pp. 467-492.

Simon Segura, Francisco (1973): La desamortización española del siglo XIX, Madrid, Instituto de Estudios Fiscales. 
Sumpsi Viñas, José Maria (1983): «El mercado y los precios de la tierra», Papeles de Economia Española, n. ${ }^{\circ}$ 16, Madrid, Confederación Española de Cajas de Ahorro, pp. 169-182.

(1986): «El mercado de la tierra y la reforma de las estructuras agrarias», Agricultura y Sociedad, n." 41, Madrid, MAPA, pp. 15-72.

Varela Ortega, Consuelo (1986 a): «Estudio econométrico sobre el mercado de la tierra en la provincia de Sevilla y Córdoba», Agricultura y Sociedad, n. ${ }^{\circ}$ 41, Madrid, MAPA, pp. 73-137.

(1986 b): «Una revisión de los modelos sobre el mercado y los precios de la tierra en la literatura económica», Agricultura y Sociedad, n. ${ }^{\circ}$ 41, Madrid, MAPA, pp. 209254.

(1988): «El mercado de la tierra en España: estructura y funcionamiento», El mercado y los precios de la tierra funcionamiento y mecanismos de intervención, Madrid, MAPA, pp. 231-270.

Zambrana Pineda, Juan Francisco (1985): «El olivar español, 1870-1930», Historia Agraria de la España Contemporánea, Vol. 2, Barcelona, Crítica, pp. 301-320. 